\title{
Digitally Assisted Preservation and Restoration of a Fragmented Mural in a Tang Tomb
}

\section{Jinglong Yang ${ }^{2} \cdot$ Jing Cao $^{1}$ (D) Haoming Yang ${ }^{3} \cdot{\text { Yuhu } \mathrm{Li}^{1} \cdot \text { Juanli Wang }}^{1}$}

Received: 12 November 2020 / Revised: 10 April 2021 / Accepted: 7 June 2021 /

Published online: 12 July 2021

(C) The Author(s) 2021

\begin{abstract}
Digital technology is being increasingly designed and employed to support the preservation and restoration of ancient murals. The contributions of digital technologies to this study were planned from the very beginning, as the complexity of this restoration necessitated the design of innovative procedures to manage the restoration and reconstruction processes and to record the details and deformation of the mural. This paper takes the digital restoration of the "maid holding a fan" mural in the Tang Zhaoling Duan Jianbi tomb as an example. In view of the large number and dislocation of mural fragments, the application of digital restoration technology and its achievements in the restoration of this ancient mural are preliminarily explored in combination with high-precision digital information collection and recording. Technical suggestions and details regarding the digital restoration of the mural are put forward to serve as guidance for the preservation and restoration of ancient murals.
\end{abstract}

Keywords Preservation and restoration $\cdot$ Mural $\cdot$ Digital restoration $\cdot$ Assisted processing

\section{Introduction}

Duan Jianbi is a part of the burial tomb of a niece of Tang Taizong Li Shimin, a dying patient in the second year of Yonghui (651); the burial tomb is approximately $5 \mathrm{kms}$ northwest of Zhaoling. In 1978, the Duan Jianbi tomb [1] was excavated and cleaned up. The object of the digital restoration described in this paper is a poorly

Jing Cao

jingcao@snnu.edu.cn

$\triangle$ Yuhu Li

liyuhu@snnu.edu.cn

$\bowtie$ Juanli Wang

wangjuanli@snnu.edu.cn

Extended author information available on the last page of the article 
preserved picture of a maid in the mural of the Duan Jianbi tomb. The mural picture is approximately $1.55 \mathrm{~m}$ long and $0.61 \mathrm{~m}$ wide. The upper half is composed of irregular fragments with obvious restoration marks, and the distribution is messy and irregular. The lines of the lower half of the picture are relatively complete, but the composition is upside down.

The digitization of cultural relics [2-8] refers to the collection and analysis of some technical information (such as shapes, lines, textures, grains, and materials) of those relics. Through the noncontact collection of data and optical measurement technology, damage to cultural relics caused by human factors in the original process of collecting information of cultural relics (prior to their protection) can be avoided. As two important noncontact methods for collecting information from ancient murals, three-dimensional and photographic technologies have been continuously improved and promoted in recent years, increasingly refining the comprehensive collection and recording of the surface information of ancient murals. From the two aspects of "colour" and "shape", these technologies can establish the information of ancient murals, and this information can become an important data basis for the protection, restoration, research and display of murals.

This paper adopts digital restoration technology to help protect and restore the "maid holding a fan" mural in the Tang Zhaoling Duan Jianbi tomb. Through the digital collection, recording and digital analysis of information regarding cultural relics, a three-dimensional digital model of the mural surface is established to help formulate a digital preservation and restoration plan [6-9]. This model can provide a data foundation and restoration support for the later restoration of the mural entity.

\section{Collection and Recording of Current Information About the Maid Mural}

\subsection{Equipment}

The equipment used for this research includes slide rails, tripods, full-frame digital cameras, microspur fixed-focus lenses, lighting assistance systems, colour correction systems, deformation control systems, etc.

\subsection{Collection of a Mural High-Definition Matrix Orthophoto}

The quality of ordinary photographic imaging is limited, and it is difficult to control the deformation, which cannot meet the strict requirements of digital preservation in the later period. Therefore, this paper adopts the high-definition matrix orthophoto collection method to record the colour information of the surface of the "maid holding a fan" mural.

The steps for collecting the high-definition matrix orthophoto (Fig. 1) include the following: 


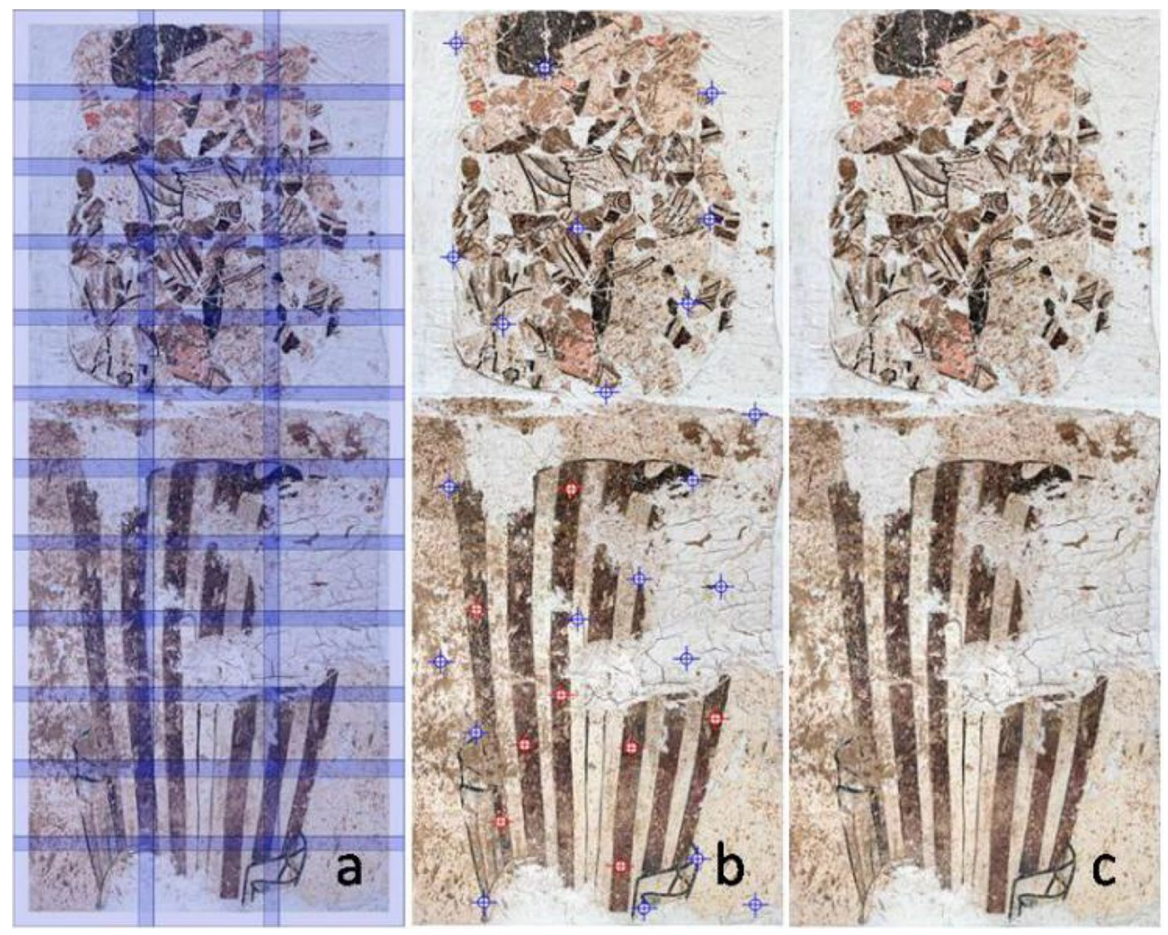

Fig. 1 High-definition matrix orthographic image of the mural a Schematic diagram of dividing the image into acquisition areas; $\mathbf{b}$ schematic diagram of the selection of control points and reference points; c. matrix orthographic image thumbnail)

1. The mural is divided into 36 areas (Fig. 1a). the ratio of the length to the width of the area is consistent with the size ratio of the sensor of the full-frame digital camera (approximately $3: 2$ ), and the overlap between each area is approximately $35 \%$.

2. The slide rails and brackets are set up, the collection distance is established according to the size of the divided block, and the colour card information in the current environment at the same time for the first collection time is recorded to check the colour later. To ensure the measurement accuracy of the digital results, 18 control points on the mural surface are selected to control the accuracy and deformation of the image, and at the same time, 8 reference points are selected on the original mural to fit the mural reference plane, which is used as the image reference plane for the mural orthophoto (Fig. 1b).

3. After the framed image is collected, the images are spliced to obtain a complete high-definition orthophoto of the mural. The image resolution is 300 DPI, the impact size is $15058 \times 38,549 \mathrm{PX}$, and the image size is $3.24 \mathrm{~Gb}$ (Fig. 1c). 


\subsection{Acquisition of High-Precision Three-Dimensional Digital Information of the Mural}

The traditional manual surveying method is to simply draw the mural. The surveying and drawing accuracy is low, and the working efficiency is extremely slow. The use of three-dimensional digital technology can quickly obtain a three-dimensional model of the mural surface, thereby accurately and scientifically recording the detailed morphological information of the mural surface. The precision of this approach is higher than that of manually drawing the mural, and the efficiency is improved.

In this paper, an industrial-grade structured-light three-dimensional scanner is used to scan the "maid holding a fan" mural surface in each area to obtain the original three-dimensional point cloud data of the mural surface (Fig. 2a), and the overlap between each area is maintained at $30-40 \%$ of the area. The three-dimensional point cloud data on the mural surface are combined, the noise are reduced, and the data are optimized to form a complete high-precision three-dimensional point cloud

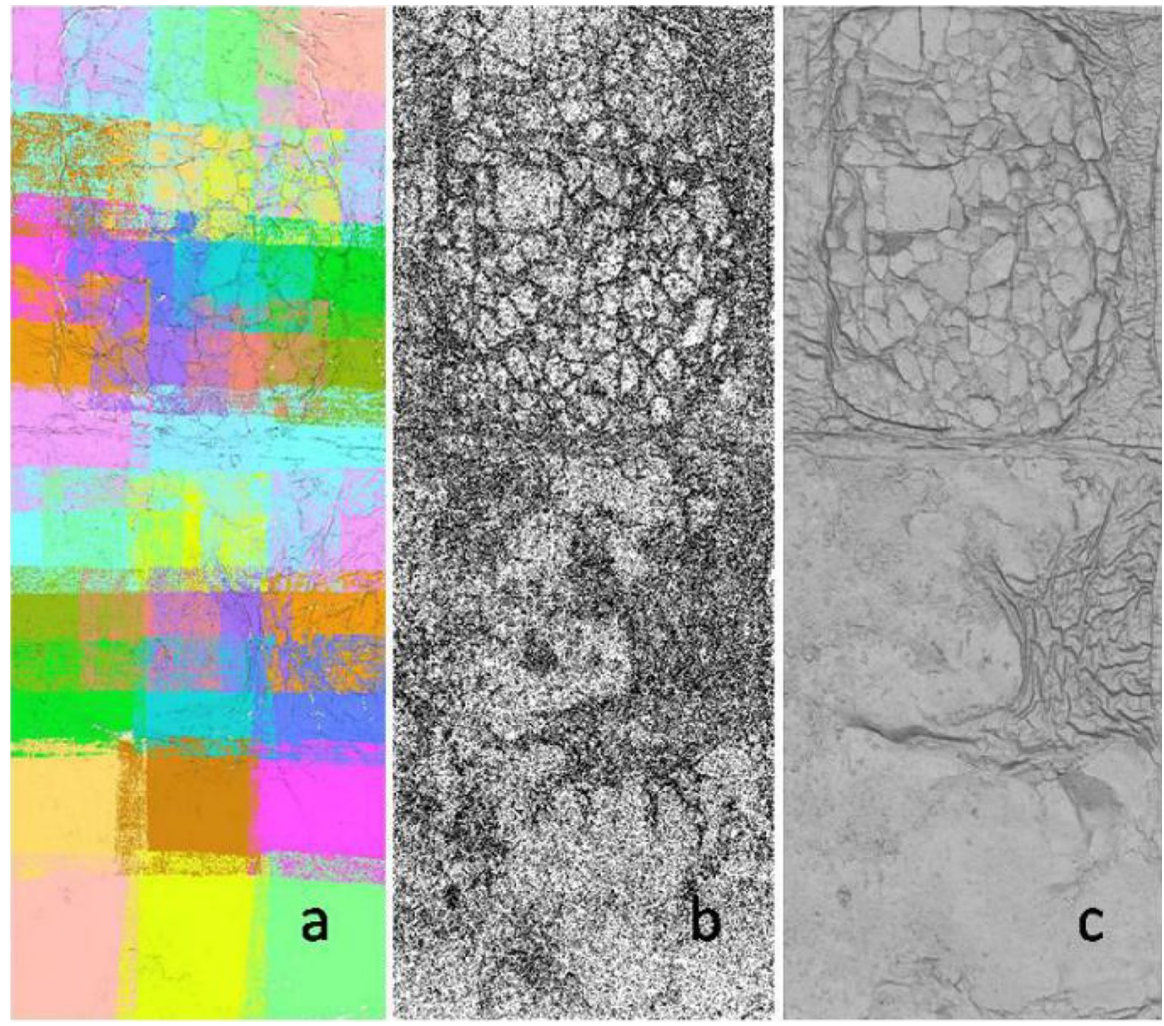

Fig. 2 Structure spectrum three-dimensional scanning diagram a Schematic diagram of the point cloud collection area; $\mathbf{b}$ complete three-dimensional point cloud data thumbnail; $\mathbf{c}$ complete three-dimensional model data thumbnail) 
(Fig. 2b). The point accuracy of the point cloud can reach $0.08 \mathrm{~mm}$, and the point spacing can reach approximately 0.20 . After the point cloud is reconstructed, a highprecision three-dimensional model of the mural surface can be obtained (Fig. 2c). The final model has a total surface area of 24.98 million pixels and a model size of $1.35 \mathrm{~Gb}$.

\section{Digital Analysis of the Maid Mural [3-5]}

\subsection{Analysis of the Status of the Maid Mural Surface}

The upper part of the "maid holding a fan" mural is basically composed of fragments (Fig. 3a), and the locations of the fragments are highly irregular. An on-site investigation revealed that the main body of the mural is composed of an aluminium alloy support, a two-layer transition layer comprising epoxy resin tape, a limestone layer, a pigment layer, and an aluminium alloy brown frame around the periphery. A surface contour image and a colour image were digitally produced on the basis of the surface topography of the mural. Due to the influence of the restoration process and the shrinkage of the lime, the debris within the historical restoration area of the mural exhibits obvious folds and grooves, the distribution angles and directions of the fragments are different, and there are a large number of areas containing debris between the fragments that need to be restored, all of which will make subsequent efforts to protect and restore the mural more difficult.
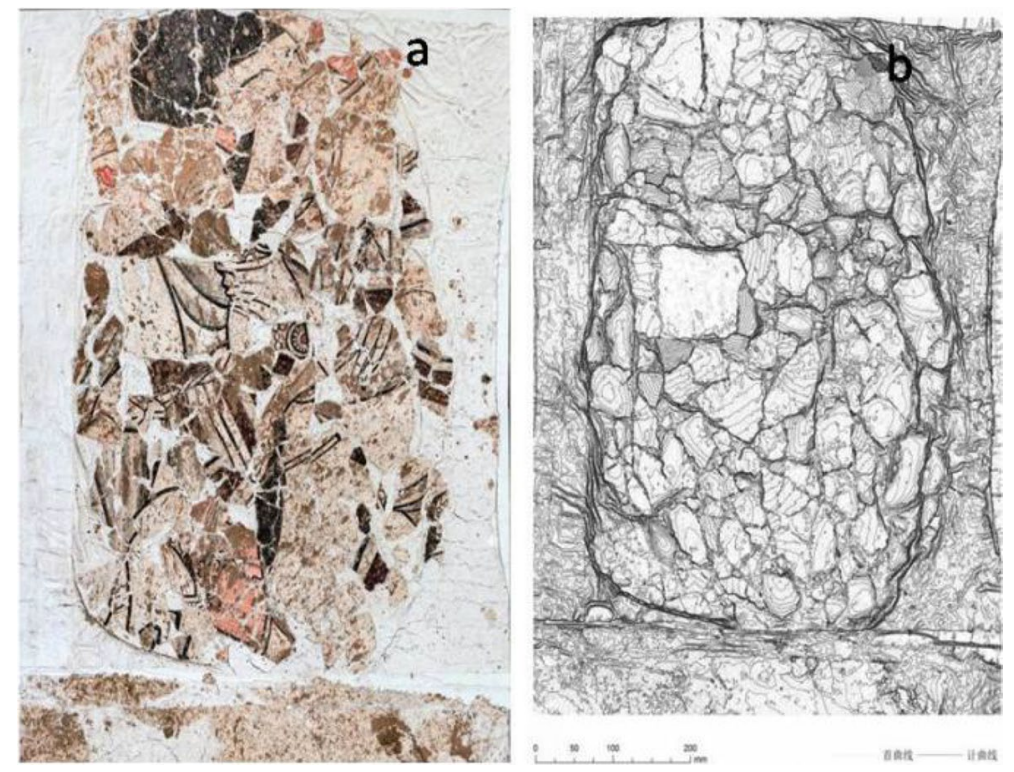

Fig. 3 Surface images of the mural a Colour image of the upper half; b surface contour image of the upper half $(0.1 \mathrm{~mm}$ contour interval)) 
The results of the above digital collection process are mainly images of the original white-grey layer and the pigment layer visible on the surface of the mural containing the colour information and three-dimensional digital topography for the first restoration of the lime layer. Based on the three-dimensional digital model of the mural surface, the surface is drawn with a $0.1 \mathrm{~mm}$ contour and equal height line chart, and the resulting image (Fig. 3b) shows the distribution of surface debris and a rough outline of the edges of the fragments.

The scattered distribution of the fragments and the irregularity of the edge contours make it clear that there are a great many fragments within the mural that will be difficult to accurately stitch together and restore. In the process of solid restoration, if the mural fragments are reset with organic adhesive, the fixation effect is poor, and the adhesive cannot easily be reversed, making it difficult to avoid secondary damage to the mural fragments if the positions and angles of the fragments are finely readjusted or reprocessed. Therefore, the mural fragments can be digitally spliced to simulate the mural fragments before the physical restoration, and this simulation can play a role in guiding the later restoration of the mural.

\subsection{Contour Extraction of the Existing Fragments of the Maid Mural}

Because the distribution of the fragments of the "maid holding a fan" mural is irregular and some areas of the mural are filled with lime needing restoration, obtaining an accurate definition of the edges of the mural has become the primary task for the digital preservation of the mural. Considering the current preservation status of the mural, this paper adopts the following methods to extract the outline of residual edges:

\subsubsection{Analysis of the Local Inclination of the Mural}

The local inclination of the mural is an important index for describing the inclination of each mural fragment. The inclination of a mural fragment is calculated by the maximum average method: the orientation of a mural fragment is the inclination of a local position in the three-dimensional model of the mural. The smaller the inclination value is, the flatter the block; the larger the inclination value is, the steeper the block.

By fitting a plane to the $\mathrm{z}$ value of a $3 \times 3$ pixel neighbourhood around the centre pixel to be processed, two parameters can be used to describe the inclination of a mural fragment: the inclination degree and the percentage elevation increment in the local inclination of the mural (Formula 1). The percentage elevation increment can be better understood by considering it as the elevation increment divided by the horizontal increment and then multiplied by 100 . When the angle is 45 degrees, the elevation increment is equal to the horizontal increment, so the percentage elevation increment is $100 \%$. When the slope angle approaches a right angle (90 degrees), the percentage elevation increment begins to approach infinity.

Formula 1: The elevation increment of the local inclination of the mural 


$$
T=\operatorname{ATAN}\left(\sqrt{[d z / d x]^{2}+[d z / d y]^{2}}\right)
$$

The results of the analysis of the surface inclination angles of the three-dimensional mural fragments are shown in Fig. 4a. The results show that the inclination angles of the fragments on the mural surface within the area of plaster restoration are mainly approximately $0^{\circ}-9.4^{\circ}$; in contrast, the inclination angles of the edges of the mural fragments and the gaps are mainly concentrated at approximately $38.5^{\circ}-85.8^{\circ}$. Hence, the edges of the mural in Fig. 4 a are orange to dark red.
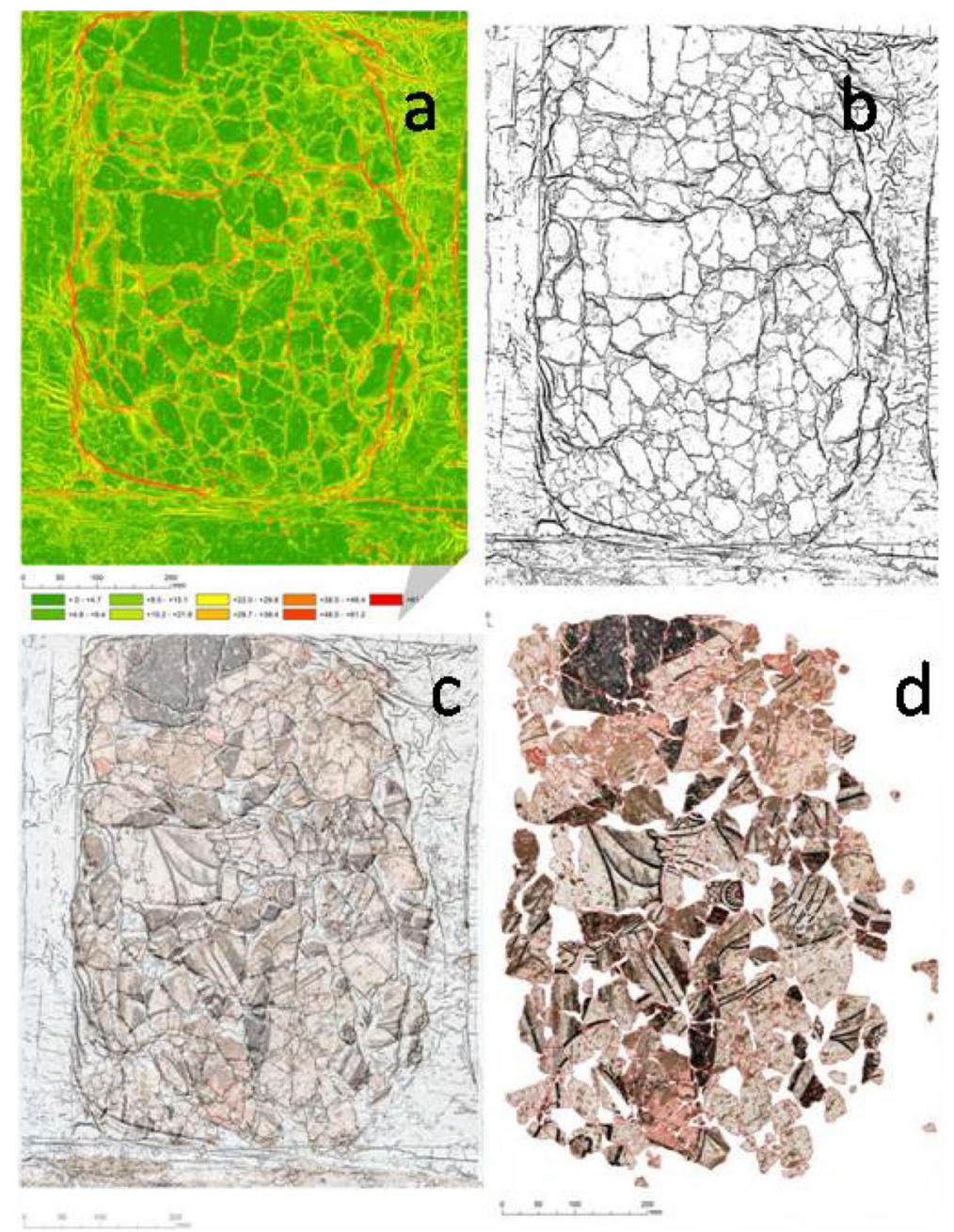

Fig. 4 Current status of the mural fragments a The results of the surface inclination analysis; $\mathbf{b}$ threedimensional point cloud showing the edges of mural fragments; $\mathbf{c}$ overlay of the positioning results and mural orthographic images; $\mathbf{d}$ edge contour information of the fragments) 


\subsubsection{Edge Analysis of Mural Fragments}

The methods for detecting edges and for extracting straight line segments from three-dimensional point clouds can be divided into two categories. The first category of methods uses the two-dimensional image corresponding to the point cloud or converts the three-dimensional point cloud into an image and then uses image processing to extract two-dimensional edges or straight line segments (which further correspond to three-dimensional point cloud data) to extract the three-dimensional edges or straight line segments; this type of method is called an "indirect method". The second type of method works directly on the threedimensional point cloud data to extract the three-dimensional edges or straight line segments, and thus, it is called the "direct method".

In this paper, the "indirect method" is used to extract the fragments outlines of the maid mural. In other words, the corresponding two-dimensional image and distance image are calculated based on the complete three-dimensional point cloud data of the mural, and the planar two-dimensional edges are monitored and classified. The obtained two-dimensional edges are superimposed onto the original three-dimensional point cloud data and then merged into multiple groups based on the edges. Along the edges of the three-dimensional point cloud of the detected mural debris (Fig. 4b), the characteristics of the debris and restored plaster edges and the gaps between the debris in the analysis results are presented as dense points.

\subsubsection{Vectorized Edge Contour Extraction of Mural Fragments}

Based on the above inclination distribution and edge analysis results, the mural edge positions are clearly visible, facilitating a small amount of manual identification and adjustment work, and the edge contours of the mural fragments can be quickly and effectively located by extracting vector lines from the dense points. Next, after overlaying the positioning results with the mural orthophoto (Fig. 4c), each mural fragment is extracted by the obtained edge contour lines. After extracting the outline information of the residual edges of the mural (Fig. 4d), a total of 283 mural fragments are obtained, including 174 ink and pigment layer fragments and 108 white-grey layer fragments.

\section{Protection and Restoration of Mural Virtual Splicing [10-14]}

Through the digital surface analysis of the maid mural and the extraction of edge contours, the edges of the fragments are determined and vectorized; the vectors extracted in this manner can be used as the basic data for virtually splicing the fragments and restoring the maid mural. Combining the colour information of the surface of the fragments, the fragments containing the picture of the mural are used as the main digital content to be spliced for protection. 


\subsection{Digital Splicing Basis}

According to the briefing report of the Tang Zhaoling Duan Jianbi tomb, there are a total of 17 existing murals, including eight maid murals. The image of the maid mural in the briefing and the digitally protected content are very consistent with the properties of aspects such as the clothing, accessories and hairstyles of the maid, and thus, these properties constitute an important basis for the restoration and splicing. In the process of splicing the fragments and for the digital preservation of the maid mural, the splicing bases are divided into the following five main categories:

\subsubsection{Features of the Picture Content}

The content of the picture is the basis of splicing together the mural fragments. However, due to the lack of original archaeological excavation data and original records containing information on the mural, it is impossible to determine the original appearance of the mural or to determine whether the existing fragments are complete. Therefore, when digitally preserving and splicing the mural fragments, first, according to the content of the picture, after a comparison with archaeological data and related research data on tomb murals from the Tang Dynasty, it can be preliminarily judged that the general content of the "maid holding a fan" mural picture is divided into the head, group fan, hand/shoulder, skirt and other parts.

\subsubsection{Drawing Technique and Workmanship}

Drawing techniques vary according to the type of paint, the width and radii of curvature of the lines, the direction of the texture, the logical relationship, etc. Digital splicing is performed strictly in accordance with the above comparison content. Any mismatch is regarded as having no correlation between the fragments. If all the pieces are matched, the result is regarded as being related to the picture, and the possible splicing position is preserved. According to the texture direction shown in Fig. 5, the original fragment states and the logical relationship between the line and texture directions before and after digital splicing are clear.
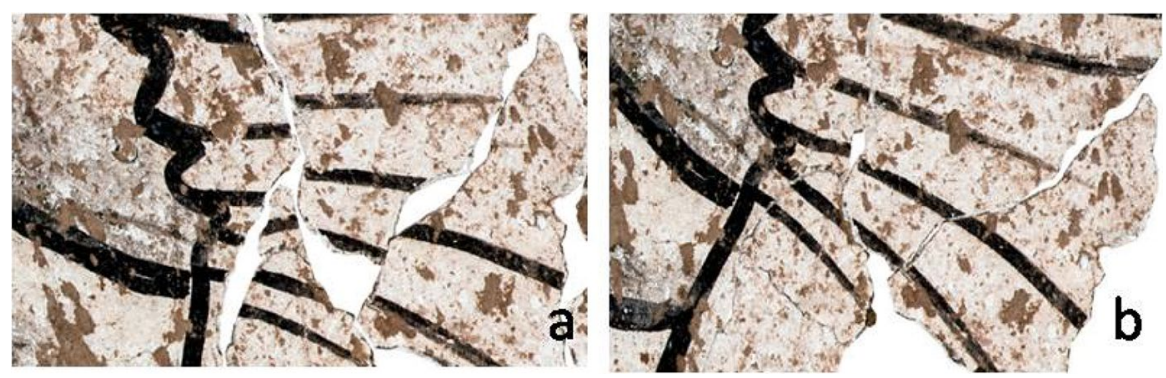

Fig. 5 Texture and line directions of the mural a original chaotic state; $\mathbf{b}$ after digital splicing) 


\subsubsection{Edge Tooth Marks}

Because the mural is fragmented, the mural comprises many irregular edges; nevertheless, the edge lines between adjacent edges have a logical relationship and interlock with each other. Therefore, the edge marks of the mural fragments can be used as another important basis for splicing the fragments. Combined with the content of the picture, the edge marks are compared, and the restoration is carried out.

As shown in Fig. 6, the red and yellow lines are the boundaries of the fragments on either side of the gap, and the edges of the fragments are fitted to each other after being traced. At the same time, the painted areas are also matched. Based on this, the fragments can be spliced.

\subsubsection{Relative Position}

Although the mural is broken, the fragments are relatively close to each other. Therefore, although the positions of the mural fragments are disturbed in the temporary reinforcement, most of the fragments are still close together, and their proximity can be used as the first reference for the first two splices.

Figure 7 depicts the head of hair of the maid in the mural. Although the fragments of the mural are disturbed and shifted, groups of fragments are relatively close to each other. Thus, it can be preliminarily determined that this group of fragments is close to the original image and can be spliced into a complete headdress.

\subsubsection{Surface Curvature}

During the preservation of the mural, due to stress, the surface is characterized by deformation composed of various curves with different radii, and the overall curvature will be discontinuous before the restoration is complete. Before the mural is
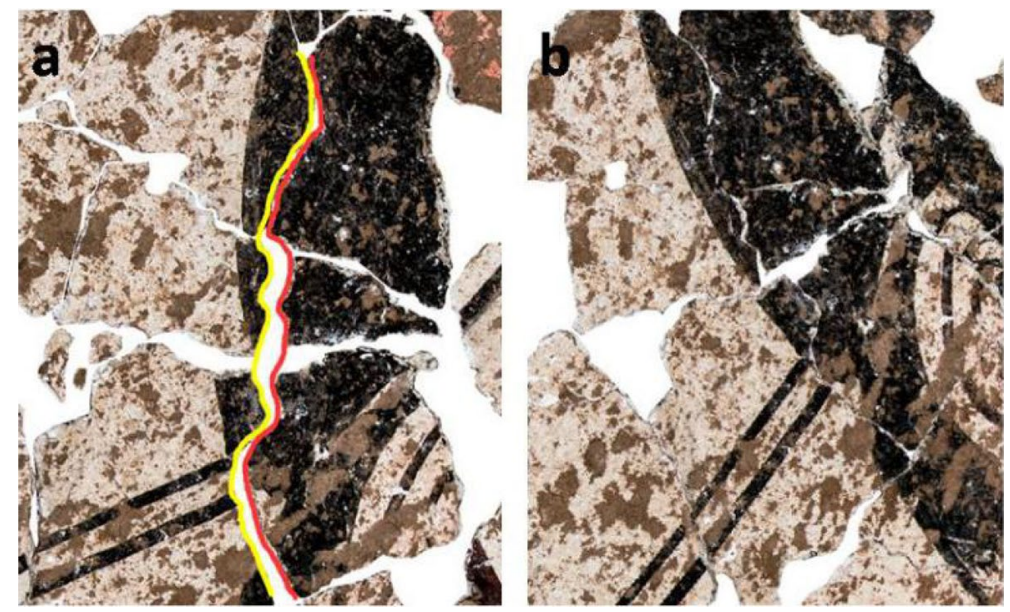

Fig. 6 Outline of the mural (a original state; $\mathbf{b}$ occlusion of contour lines) 
Fig. 7 Relative positions of mural fragment edges

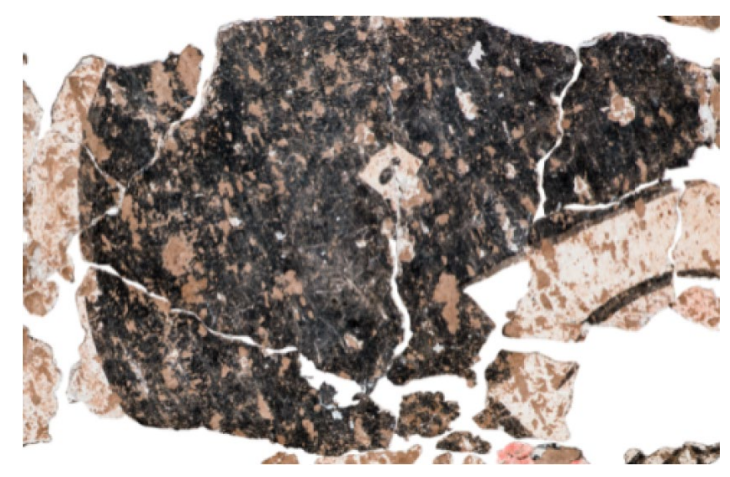

restored, high-definition photographic images and three-dimensional digital information of the mural fragments are collected. Referring to the results of the previous digital restoration and splicing steps, after the physical restoration is completed and after the mural fragments are returned to their original positions, thereby completing the mural, a curvature comparison can be conducted to verify the accuracy of the restored mural fragment positions.

\subsection{Digital Preservation Splicing Results}

Due to the large amount and size of debris and the complex tooth marks on the edges of the mural, it is difficult to quickly compare and mosaic the debris together. Therefore, it is necessary to group the mural into areas (head, fan, shoulders, skirt, etc.) based on the lines within the debris and the characteristics of the paint. At the same time, each residual block is independently numbered, corresponding translations and rotations are performed according to the abovementioned splicing basis, and the scale of the residual block is preserved. The fragments are restored one by one, and the angles and positions of the local fragments are finely adjusted. The final results of the digitally reconstructed mural are shown in Fig. $8 \mathrm{a}$ and b. There are 160 fragments based on direct splicing; however, there are 14 fragments containing ink lines or paint layers that cannot be accurately connected to the main body of the picture, and 108 fragments of the white-grey layer are also not included in this splicing work.

\subsection{Mural Restoration and Line Drawing Restoration}

After the initial digital reconstruction of the "maid holding a fan" mural, due to the lack of mural fragments, the subsequent completion of the mural is based mainly on harmonic colours and smooth lines. The evidence-based lines are completed based on their width, radii of curvature, and logical relationships. At the same time, according to the digitally restored image of the mural, drawing the lines of a female figure can be reasonably justified (see Fig. 8c). The digitally restored image and line restoration of the mural can provide an important reference basis for subsequent splicing and restoration of the mural entity. 

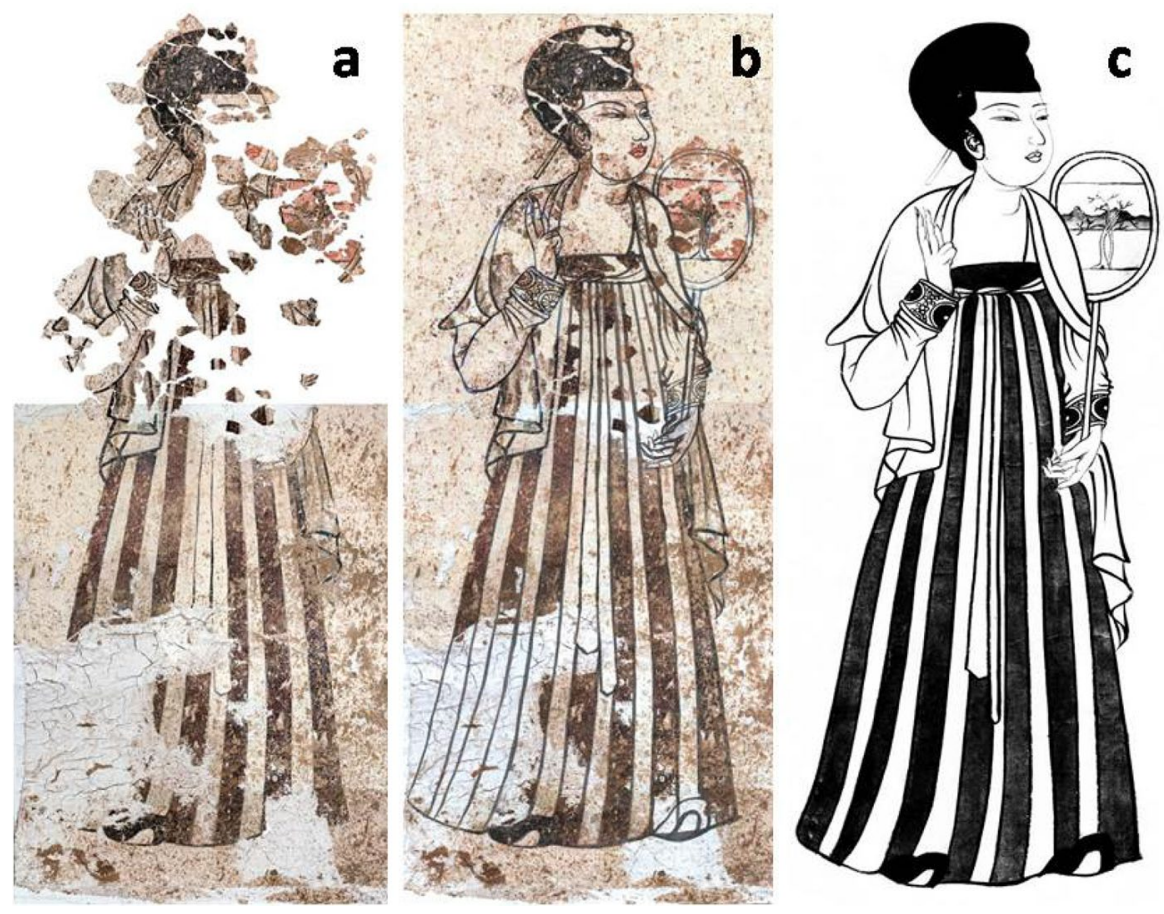

Fig. 8 Digital restoration effect a Direct effect of digital splicing; b digital splicing restoration effect; c restored mural image)

\section{Mural Entity Restoration}

The "maid holding a fan" mural was initially restored after being unveiled in 2002. Since the mural artwork covered with peach tape has not been protected, the upper part of the mural (after removing the peach tape) was found to be severely broken and poorly preserved, basically composed of mural fragments, the positions of which are irregular. Therefore, the first restoration focused mainly on salvaging fragments and removing debris and temporarily fixed mural fragments. The specific steps were as follows: limestone layer $\rightarrow$ epoxy resin tape reinforcement $\rightarrow$ aluminium alloy frame as a support $\rightarrow$ peeling off the peach tape $\rightarrow$ adding brown aluminium edging for decoration $\rightarrow$ retaining the data. The restored mural is shown in Fig. 1.

On the basis of the previous digital restoration, according to the traditional mural restoration process, the mural entities were comparatively restored. 


\subsection{Comparative Restoration of the Mural Entities}

\subsubsection{Printing the Digitized Lines of the Restored Mural onto a Water Transfer Sticker [15]}

The restoration adopted the restored mural lines shown in Fig. 8c and the contour outline of the debris on the upper half of the mural (Fig. 3b) obtained by the previous digitization using a water transfer surface treatment. The medium was made of water transfer paper [15], environmental protection ink, environmental protection glue and lamination printing. The lines of the restored mural printed onto a water transfer sticker and the water transfer sticker of mural fragments were ready for use.

\subsubsection{Removal of the Mural Limestone Layer and Keel}

During the restoration, the front of the mural was protected with silicone sponges and wooden boards. After turning the mural over, the limestone layer and keel support left from the back of the mural were removed for the first restoration (Fig. 9a), and the back of the dust and debris were thoroughly cleaned.

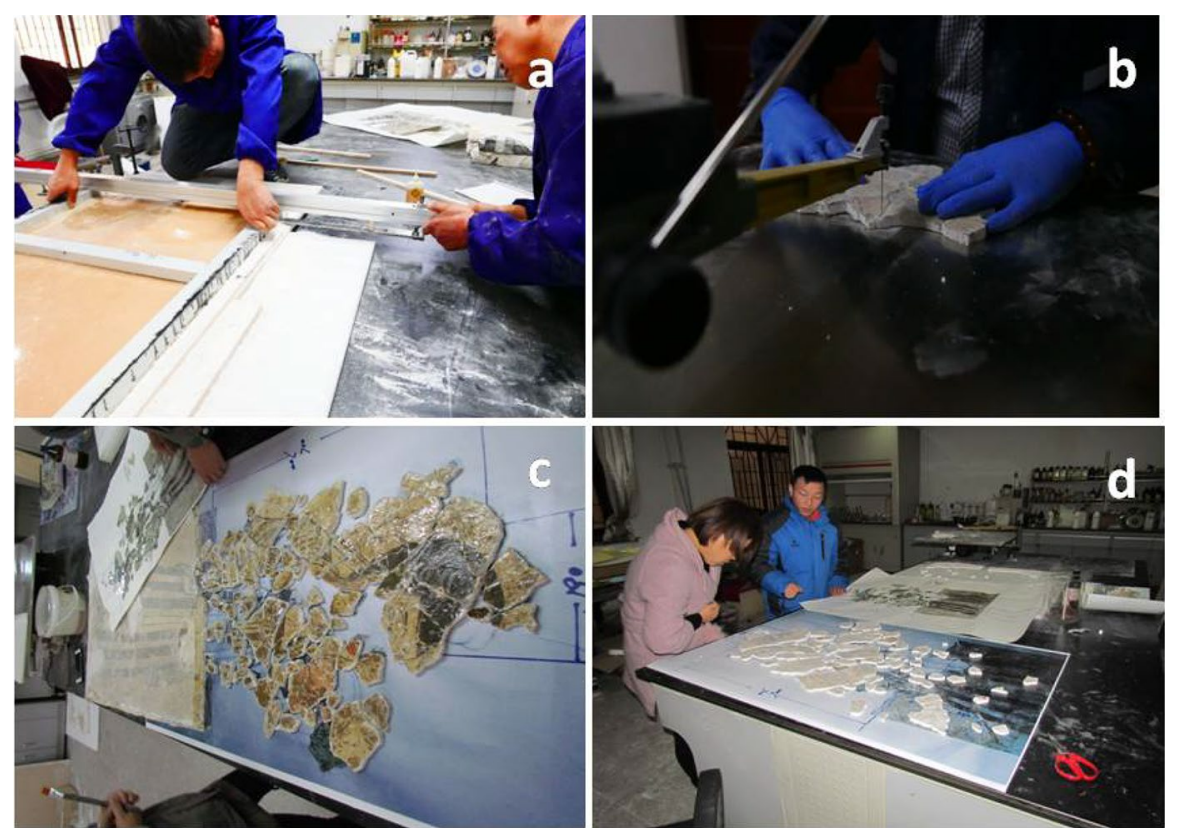

Fig. 9 Entity restoration steps (a Removing the limestone layer and keel; b cutting the fragments; c marking the line blocks; d splicing.) 


\subsubsection{Cleaning the Mural Surface}

The mural was turned over to gain access to the front, floating dust on the surface of the mural was gently removed with a soft brush, and then a cotton swab dipped in deionized water was used to remove any dust and contaminants adhered to the mural surface.

\subsubsection{In Situ Superimposition of the Contour Map of the Debris onto the Upper Half of the Mural}

After a thorough cleaning, a cotton swab dipped in a small amount of deionized water was taken to the mural surface. After wetting the outline of the debris on the upper half of the printed mural lightly, the contour lines and key parts of the debris on the upper half of the mural were compared. The texture was accurately superimposed onto the lines and then posted onto the surface of the mural in situ, and the contour map was pressed gently to eliminate the air between the sticker and the mural for a close fit. After $2 \mathrm{~min}$, the water transfer sticker was slowly removed, and the outline of the edge of the upper part of the mural was transferred from the water transfer sticker to the surface of the mural.

\subsubsection{Cutting the Fragments According to the Contours of the Fragment Edges}

The contour lines of the edges of the mural were cut (as shown in Fig. 9b), and the mural with the printed outline from the digitally reconstructed mural was also cut. The positions of the fragments were placed one by one (Fig. 9c and d).

\subsubsection{Making a New Limestone Layer}

A soft bristle brush was used to evenly dip 5\% hydroxy cellulose solution onto the back of the well-matched mural fragments so that it could be closely affixed with sulfuric acid paper (as shown Fig. 10a) while referring to the restored mural line diagram. The lower part of the mural was accurately matched, and the fragments were fixed after adjusting their positions. Then, the mural was flipped, and a new limestone layer was made with lime plaster and a hemp knife (as shown in Fig. 10b).

\subsubsection{Restoration of Mural Picture Layer}

The mural was flipped back over again, the sulfuric acid paper was peeled off, and the limestone layer of the new mural was manually thinned by 1-2 mm. Combined with archaeological excavation data and previous digital restoration results, the splicing lines on the upper half of the mural were traced onto the surface of the new limestone layer in accordance with the traces, and the old sections with no paint layer were processed. The restored mural fully displays the original style 

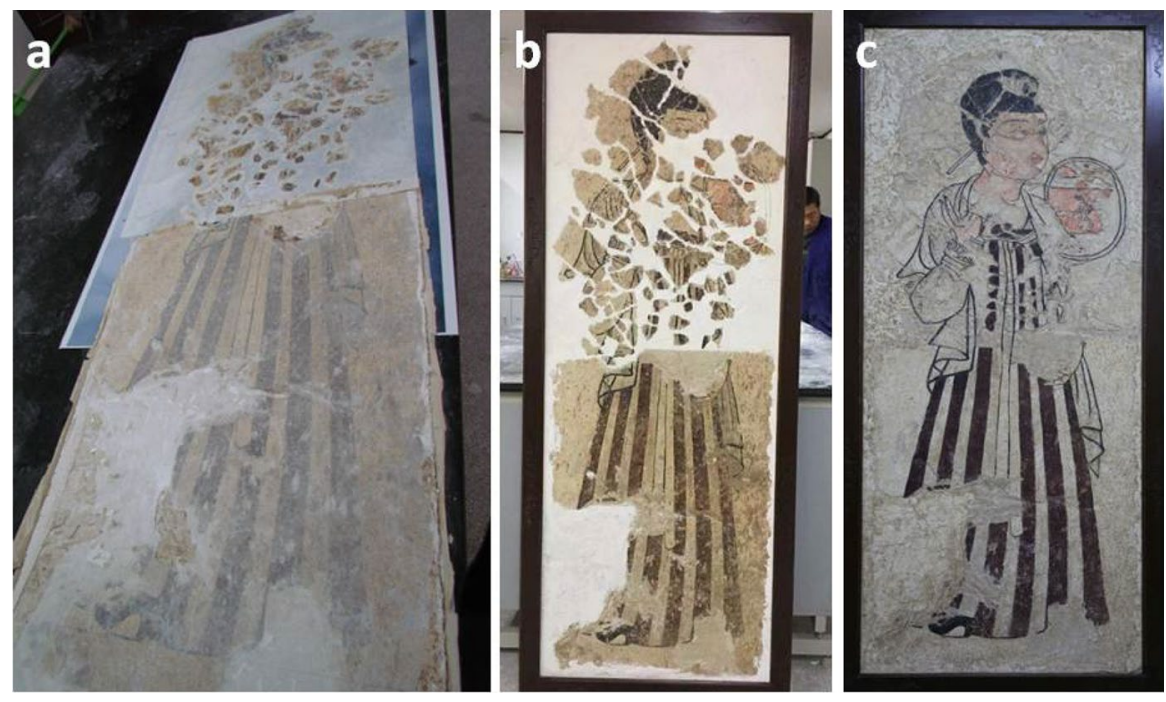

Fig. 10 Restored mural entity (a Restoration of the picture layer; b In situ superimposition; $\mathbf{c}$ restored mural)

and artistic integrity of the original picture, although traces of the restoration can still be distinguished when viewed closely (as shown in Fig. 10c).

\section{Conclusion}

1. The high-definition digital orthophoto collection technology and high-precision three-dimensional digitation technology are innovative and mature techniques for restoring murals, as they can collect the "shape" and "colour" information from the mural surface with high efficiency and low cost. These technologies are the best means to collect and record the surface information of a mural.

2. Based on a surface analysis of the high-precision three-dimensional model of the mural, the deformation of the mural, such as along the edges of the mural fragments, crack positions, and folding, can be analyzed and quantified. Based on a high-definition orthophoto of the mural, auxiliary digital preservation and planning can be carried out, virtual restoration can be performed, and colour anomalies can be investigated and quantified.

3. Based on the high-precision three-dimensional model of the mural, the edge contours of the mural fragments were extracted and superimposed onto the mural high-definition orthophoto, and an image of the mural fragments was extracted, which was supplemented by a small amount of manual picture screening work. This approach can quickly and efficiently extract the mural fragments and the outline of the edges to facilitate the digital preservation and splicing of the mural painting and other subsequent work. 
4. According to the 5 kinds of mural fragment splicing bases summarized in this paper, the mural fragments (except for those in the white-grey layer) were stitched together reasonably and accurately, but due to the large area missing from the mural, a small number of isolated fragments still lacked an accurate splicing basis, and thus, precise digital preservation and splicing could not be carried out.

5. On the basis of adhering to the traditional restoration technology, especially for severely damaged murals for which the positioning of noncontinuous lines and sites from lines and textures cannot be achieved, digital restoration is used as preliminary guidance for auxiliary mural restoration through the use of digital simulation stitching at spatial sites. In this way, an effective digital restoration can be simulate, and restoration line drawings can be established, which can be used as the basis for a physical restoration and to optimize the restoration of the mural to its original appearance in combination with archaeological data.

6. A water transfer surface treatment was used in the physical restoration, which is convenient for superimposing the mural surface in situ. In this technique, the restored mural lines are placed along the ink lines of the water transfer sheet. On the one hand, the lines are distinguished from the original lines of the mural screen; on the other hand, the water transfer sheet is easy to remove. The restored mural can not only fully show the style and artistic integrity of the original picture but also enable the minimal amount of intervention in the restoration of cultural relics.

In view of the existence of a large number of fragments, similar to the physical restoration and protection of the "maid holding a fan" mural or other nearly planar but broken cultural relics, there is no reasonable scientific prediction and restoration plan for splicing the fragments together; in this case, secondary injury to the mural may occur. It is recommended that the surface information of cultural relics be collected under noncontact conditions, that the restoration process be simulated, and that a digital restoration be conducted through digital means to serve as an important guide and reference for subsequent physical restoration. This approach will ensure that unnecessary secondary damage is reduced during the restoration of the entity and that the authenticity of the restored entity remains intact.

Acknowledgements The authors would like to thank the Xianyang City Cultural Relic Protection Center and Fire Companion Art Culture Technology Co., Ltd., Xi'an, for their support and research cooperation.

Authors' Contributions All authors contributed to the study concept and design. All authors commented on previous versions of the manuscript. All authors read and approved the final manuscript. JLY conceived the research and conducted the on-site data collection and the preservation and restoration processing of the ancient mural in accordance with the research methods. JC designed the research methodology, performed the experiments, data acquisition and processing, and drafted the manuscript. HMY performed analysis of the part of experimental data. YHL guided the preservation and restoration processing and reviewed the manuscript. JLW guided the design of the research methodology, analysis of the experimental data, research application and revision of the manuscript.

Funding The authors gratefully acknowledge the financial support received by the Fundamental Research Funds for the Central Universities (No. GK 20210361, GK 202103058). 
Data Availability The datasets used and/or analysis results obtained in the current study are available from the corresponding author on request.

\section{Declarations}

Conflict of interests The authors declare that they have no conflicts of interest related to this work. The authors declare that they do not have any commercial or associative interest that represents a conflict of interest in connection with this work submitted.

Open Access This article is licensed under a Creative Commons Attribution 4.0 International License, which permits use, sharing, adaptation, distribution and reproduction in any medium or format, as long as you give appropriate credit to the original author(s) and the source, provide a link to the Creative Commons licence, and indicate if changes were made. The images or other third party material in this article are included in the article's Creative Commons licence, unless indicated otherwise in a credit line to the material. If material is not included in the article's Creative Commons licence and your intended use is not permitted by statutory regulation or exceeds the permitted use, you will need to obtain permission directly from the copyright holder. To view a copy of this licence, visit http://creativecommons.org/licen ses/by/4.0/.

\section{References}

1. Ge, H., Li, Y. H., Wang, J. L., (2018) Study on the pre-reinforecement and soil rust removing technology of the maid figure from the TANG Zhaoling Duan Jianbi tomb murals, 46(2), 68-73.

2. Bao, A. D., Chen, C., \& Zheng, Y. (2017). The practice and discovery of digital protection in the investigation of shuanglin temple color and plastic mural painting [J]. Chinese Cultural Heritage, 05, 40-50.

3. Wei, H., Hu, Y. G., \& Hou, M. L. X. D. (2017). Zhang research on the method of dividing the mural photos into blocks [J]. Geographic Information World, 24(03), 107-112.

4. Xin, N. (2016). Wisdom on fingertips-Tibet mural art and digital protection and restoration [J]. Chinese National Art, 01, 72-77.

5. Xie, H. Y. (2013). Briefly describe the key technical process of digitalization and reductive reproduction of cave paintings [J]. Digital Printing, 3(03), 31-33.

6. Yu, T. X., Wu, J., Sun, Z. J., Zhao, L., \& Ding, X. H. (2011). Research on image splicing method of Mogao Grottoes digital fresco based on improved geometric deformation [J]. Dunhuang Research, 06, 91-95.

7. Wang, J. R. (2011). Digital collection technology and application of high-precision image of murals in Tang Dynasty [J]. Wenbo, 03, 66-70.

8. Pan, Y. H., \& Lu, D. M. (2003). Digital protection and restoration of ancient Dunhuang frescoes [J]. Journal of System Simulation, 03, 310-314.

9. Zheng, Y., (2015) Digital Technology in the protection of cultural heritage Bao Fan Temple mural digital mapping survey[J]. ISPRS - International Archives of the Photogrammetry, Remote Sensing and Spatial Information Sciences, XL-5/W7(1): 495-501.

10. Vanezis, P., Blowes, R. W., Linney, A. D., Tan, A. C., Richards, R., \& Neave, R. (1989). Application of 3-D computer graphics for facial reconstruction and comparison with sculpting techniques[J]. Forensic SciInt., 42(1-2), 69-84.

11. Rusine, K. H., Noz, M. E., Maguire, G. Q., et al. (1991). Quantitative and qualitative comparison of volume tricand surface rendering techniques[J]. IEEE Transaction Nuclear Science, 38(2), 659-662.

12. Pei, S. C., Zeng, Y. C., \& Chang, C. H. (2004). Virtual restoration of ancient Chinese paintings using color contrast enhancement and lacuna texture synthesis[J]. IEEE Transactions on Image Processing. https://doi.org/10.1109/TIP.2003.821347

13. Wang S. W., XU Y. S., (2009) Improvement of priority computation in exemplar-based image inpainting based on D-Sevidence theory and TV model[J]. Proceedings of the 2nd International Congress on Image and Signal Processing. 
14. Criminisi, A., Perez, P., \& Toyama, K. (2004). Region filling and object removal by exemplar-based image inpainting[J]. IEEE Transactions on Image Processing. https://doi.org/10.1109/TIP.2004. 833105

15. Zou, Y., \& Zhang, Y. F. (2009). The Fundamentals of Water Transfer and Procedures Production[J]. Screen Printing, 5, 38-40.

Publisher's Note Springer Nature remains neutral with regard to jurisdictional claims in published maps and institutional affiliations.

\section{Authors and Affiliations}

\section{Jinglong Yang ${ }^{2} \cdot$ Jing Cao $^{1}$ (D) Haoming Yang ${ }^{3} \cdot{\text { Yuhu } \mathrm{Li}^{1} \cdot \text { Juanli Wang }}^{1}$}

Jinglong Yang

512552996@qq.com

Haoming Yang

twpqeqr0226@163.com

1 Engineering Research Center of Historical and Cultural Heritage Protection, Ministry of Education, Shaanxi Normal University, No.620, West Chang'an Street, Xi'an City, Shaanxi Province, China

2 Xianyang Heritage Conservation Center, Baoquan Street, Xianyang City, Shaanxi Province, China

3 East China University of Science and Technology, Haiwan Zhenhai Train of Thought, No. 999, Fengxian District, Shanghai, China 